\title{
Simple Preoperative Patient-Reported Factors Predict Adverse Outcome After Elective Cranial Neurosurgery
}

\section{Reponen, Elina}

2018

Reponen , E , Korja , M \& Tuominen , H 2018 , ' Simple Preoperative Patient-Reported Factors Predict Adverse Outcome After Elective Cranial Neurosurgery ' , Neurosurgery (Baltimore), vol. 83 , no. 2 , pp. 197-202 . https://doi.org/10.1093/neuros/nyx385

http://hdl.handle.net/10138/312066

https://doi.org/10.1093/neuros/nyx385

unspecified

acceptedVersion

Downloaded from Helda, University of Helsinki institutional repository.

This is an electronic reprint of the original article.

This reprint may differ from the original in pagination and typographic detail.

Please cite the original version. 
1 Simple preoperative patient-reported factors predict adverse outcome after elective cranial

2

3 Elina Reponen, $\mathrm{MD}, \mathrm{PhD}^{1}$ Miikka Korja, $\mathrm{MD}, \mathrm{PhD}^{2}$ Hanna Tuominen, $\mathrm{MD}, \mathrm{PhD}^{1}$

4

$5 \quad{ }^{1}$ Department of Anaesthesiology, Intensive Care and Pain Medicine, University of Helsinki and

$6 \quad$ Helsinki University Hospital, Helsinki, Finland

$7 \quad{ }^{2}$ Department of Neurosurgery, University of Helsinki and Helsinki University Hospital, Helsinki, 8 Finland

9

10

11

12

13

14

Correspondence: Dr. Elina Reponen at the Department of Anaesthesiology, Intensive Care and Pain Medicine, Helsinki University Hospital, P.O. Box 900, FI-00029 Helsinki, Finland; E-mail address: elina.reponen@hus.fi; Telephone: +358 50427 9469; FAX: +358 947187721.

Funding: This work was supported by personal grants to E.R. from the Finnish Medical Association and the Finnish Society of Anaesthesiologists.

\section{Details of author contributions}

E.R.: Study design, patient recruitment, data collection, data analysis, and writing up of the first draft of manuscript.

M.K.: Study design, data analysis, and manuscript preparation.

H.T.: Study design, patient recruitment, and manuscript preparation.

\section{Disclosure/Conflicts of Interest}

None declared. 


\section{ABSTRACT}

\section{Background}

3 Patient-reported preoperative factors hold promise in improving the prediction of

4 postoperative adverse events, but they have been poorly studied.

\section{Objective}

6 We aimed to study the role of patient-reported factors in the preoperative risk stratification of

7 elective craniotomy patients.

\section{Methods}

9 A prospective, unselected cohort of 322 adult patients underwent elective craniotomy in XX.

10 We preoperatively recorded the American Society of Anesthesiologists (ASA) score, Helsinki

11 ASA score, and three questionnaire-based patient-reported factors including overall health

12 status, ability to climb two flights of stairs, and cognitive function [Test Your Memory

13 (TYM) test]. Outcome measures comprised in-hospital major and overall morbidity. Receiver

14 Operating Characteristic (ROC) curves served to calculate Area Under the Curve (AUC)

15 values for a composite score of patient-reported factors and both ASA scores with regard to

16 outcomes.

\section{Results}

In-hospital major and overall morbidity rate was $15.2 \%$. Only preoperatively diminished cognitive function remained a significant predictor of major morbidity after multivariable logistic regression analysis ( $<<0.001$, OR 1.1, CI 1.0-1.1). A composite score of our three

21 patient-reported factors had a higher AUC (0.675) for major morbidity than original ASA

22 score (0.543) or Helsinki ASA score (0.572). In elderly patients, the composite score had an

23 AUC of 0.726 for major morbidity.

\section{Conclusions}

25 Preoperative patient-reported factors had higher sensitivity for detecting major morbidity compared to the ASA scores in this study. Particularly the simple composite score seems to 
27 predict adverse outcomes in elective cranial surgery surprisingly well, especially in the 28 elderly. These results are interesting and worth confirming in other centers.

29 Running title: Patient-reported preoperative factors and craniotomy outcome

30 Key words: craniotomy, outcome, patient-reported, preoperative risk assessment 


\section{INTRODUCTION}

33 Preoperative risk assessment is considered a routine preparation for major surgery worldwide.

34 Some surgical specialties, including cardiac surgery, have developed tailored risk scores for

35 customized risk stratification in specific patient groups. ${ }^{1,2}$ For many types of major surgery,

36 including neurosurgery, only more generic risk assessment scores are available. They are

37 usually based on the burden of comorbidity, ${ }^{3-5}$ functional status, ${ }^{6-8}$ or focus on individual

38 organ systems. ${ }^{9,10}$ The role of conventional preoperative risk assessment scores in elective

39 cranial neurosurgery is unclear and the evidence is scarce. ${ }^{11}$

40 Patient-reported outcomes are increasingly used for surgical outcomes reporting. Patient-

41 centered care calls for more direct patient involvement also in the surgical decision-making

42 process. Little evidence, however, exists on the use of patient-reported factors in preoperative

43 risk stratification, especially in neurosurgery. In this prospective cohort of adult elective

44 craniotomy patients, we compared the risk-predicting ability of patient-reported preoperative

45 variables with the most widely used preoperative risk score, the American Society of

46 Anesthesiologists (ASA) physical status score ${ }^{3}$ and its local modification, the Helsinki ASA

47 score. ${ }^{12}$ We aimed to study the possible benefits of including patient-reported variables in the

48 preoperative risk stratification for short-term adverse outcomes in elective cranial

49 neurosurgery. 
METHODS

\section{Ethics approval}

This study received approval from the Ethics committee of XX. A written informed consent was given by all study patients before enrollment in the study. The study was observational and all patients received preoperative, intraoperative and postoperative care according to the standard clinical practice in our department.

\section{Study population}

We have described the study protocol in previous articles based on the same cohort. ${ }^{12-14} \mathrm{~A}$ study enrollment flow-chart was presented in a previous article. ${ }^{12}$ Adult patients ( $\geq 18$ years) fluent in Finnish or Swedish with any indication for elective craniotomy except for epilepsy were eligible to participate in the study. Patients unable to communicate due to severe underlying illness or advanced cognitive dysfunction were excluded. In brief, 551 consecutive patients undergoing elective cranial neurosurgery for in XX university Hospital between Dec 7, 2011 and Dec 31, 2012 were considered eligible. The lack of obtaining informed consent prior to surgery led to the exclusion of 85 patients, and 47 refused to participate. One patient withdrew consent during the study. Thus, the original cohort comprised 418 (75.9\%) of the 551 eligible patients. Complete data for all patient-reported factors, preoperative ASA score, Helsinki ASA score, and all study outcomes were available for 322 patients who were thus included in the current analyses.

\section{Preoperative patient-reported factors}

Preoperative consultations with a neuroanaesthesiologist took place either at the preoperative outpatient clinic (one week) or at the neurosurgical ward (one day) before the scheduled surgery. At the time of preoperative consultation, an anesthesiologist or a preoperative clinic nurse asked the patient to fill in a questionnaire. Patients reported all factors on paper questionnaires created for the purposes of this study. The questions on health-related habits such as exercise habits and stair climbing were adapted from the Health 2000 study of the National Public Health Institute in Finland. ${ }^{15}$ The questionnaire included three preoperative patient-reported factors. First, the patients reported whether they were able (yes or no) to 
climb two flights of stairs without resting. In Finland, a flight of stairs is defined as a vertical climb between two floors, which equals to at least 4 vertical meters. The stair-climbing test is reliable in assessing cardiorespiratory fitness, ${ }^{16}$ and it is frequently used for preoperative risk evaluation in thoracic surgery. ${ }^{17}$ Second, the patients categorized their subjective overall health status into five categories: excellent, good, average, poor or very poor. Third, the Test your memory (TYM) -questionnaire provided a measure of the patients' preoperative cognitive function. A TYM score of $\leq 44$ points correctly identifies $96 \%$ of patients with mild Alzheimer's disease, whereas a score of $\geq 45$ implies normal cognitive status. ${ }^{18}$ Furthermore, the consulting anesthesiologist recorded the preoperative original ASA score and Helsinki ASA score ${ }^{12}$ at the time of preoperative consultation.

\section{Study outcome}

The primary outcome was major morbidity as previously described. ${ }^{13,14}$ In brief, major morbidity was defined as at least one of the following: new or worsened hemiparesis, silent stroke, pneumonia, acute myocardial infarction (AMI), deep venous thrombosis (DVT), pulmonary embolism (PE), re-craniotomy/endovascular intervention, or in-hospital mortality. Morbidities were recorded at any time during the in-hospital period except for new or worsened hemiparesis, which was recorded at hospital discharge to exclude transient neurological deficits that resolved before discharge. Reoperations were recorded up to 30 postoperative days. Hospital databases and the Population Register Center of Finland provided in-hospital mortality rates.

\section{Composite score of significant patient-reported factors}

To evaluate the benefits of using patient-reported factors in the preoperative risk assessment of elective craniotomy patients, we constructed a simple unweighted composite score. We included all patient-reported factors with significant associations with major morbidity: Poor preoperative overall health status, inability to climb two flights of stairs, and preoperatively diminished cognitive function. One point was scored for each. Thus, scores ranged from 0 to 3.

\section{Statistical analyses}


106 For statistical analyses, subjective overall health score was dichotomized as good (average,

107 good, or excellent) or poor (poor or very poor) as the classification is not ordinal. Pearson

108 Chi-square test or Fisher's Exact test enabled studying correlations for categorical variables

109 (stair climb, overall health) and Mann-Whitney U test was used for continuous or ordinal

110 variables (TYM score, ASA score, Helsinki ASA score) in relation to dichotomized outcome

111 in univariable analyses. Where applicable, we calculated odds ratios (ORs) and 95\%

112 confidence intervals (CIs) for significant factors. In all tests, $p$-value $<0.05$ was considered

113 significant. We used a multivariable logistic regression model including all significant factors

114 in univariable analyses (poor overall health, inability to climb two flights of stairs, and

115 diminished cognitive function) to identify independent outcome predictors. Hosmer and

116 Lemeshow test served for estimating the goodness-of-fit of our model. We used Receiver-

117 Operating Characteristic (ROC) curves to calculate the Area Under the Curve (AUC) values

118 to compare the predictive ability of our simple composite of patient-reported preoperative

119 factors and both the original ASA score and the Helsinki ASA score. The IBM SPSS 21.0

120 statistical software version for Windows was used in all statistical analyses. 


\section{RESULTS}

123 The demographic patient characteristics and surgical indications for the original cohort and

124 the subgroup included in the analyses for patient-reported preoperative factors are very

125 similar indicating no apparent selection bias; only the proportion of malignant and benign

126 tumors as surgical indication showed a small difference between the two groups (Table 1).

127 Of 322 respondents, only 41 (12.7\%) patients reported inability to climb two flights of stairs.

128 A majority of patients $(289,89.8 \%)$ reported a good subjective preoperative overall health. A

129 total of $101(31.4 \%)$ scored less than 45 points in the TYM questionnaire suggesting a

130 diminished cognitive function. Only $5.6 \%$ of the patients had a preoperative original ASA

131 score $>3$, and a majority (86.6\%) had preoperative Helsinki ASA score $\leq 3$ (Table 2). The

132 distribution of the preoperative patient-reported composite score is presented in Table 2.

\section{In-hospital major morbidity}

134 Major in-hospital morbidity (including mortality) was recorded in $15.2 \%$ of patients. The

135 frequencies of individual major complications in this subgroup are presented in Table 3.

\section{Univariable analysis}

137 Poor preoperative overall health status, inability to climb two flights of stairs, and

138 preoperatively diminished cognitive function were associated with objective in-hospital major

139 morbidity unlike original ASA score or Helsinki ASA score (Table 4). Major morbidity rate

140 was considerably higher in elderly patients with cognitive dysfunction, as $12(34.3 \%)$ of 35

141 patients with a combination of age 65 years or older and preoperatively diminished cognitive

142 function (TYM score <45) (sensitivity 80.0\%, specificity $64.1 \%$, PPV $34.3 \%$ and NPV

$14393.2 \%$ ) had major complications. Only 18 (10.2\%) out of 177 patients with a combination of

144 age less than 65 years and preoperatively good cognitive status (TYM score 45 or more)

145 suffered from major morbidity.

146 Multivariable analysis 
147 Of the three patient-reported factors, only preoperatively diminished cognitive function

148 remained a significant predictor of major morbidity after multivariable logistic regression

149 analysis ( $\mathrm{p}<0.001$, OR 1.1, CI 1.0-1.1, Hosmer and Lemeshow 0.325).

$150 \quad$ ROC and AUC

151 The AUC of both the original ASA score (0.543) and Helsinki ASA score (0.572) for major

152 morbidity were low. The composite score AUC for major morbidity was 0.675 (Figure 1).

153 In the subgroup of elderly patients, the original ASA score had AUC 0.532 and Helsinki ASA

154 score had AUC 0.511 for major morbidity. The AUC of the composite score in this subgroup 155 was 0.726 . (Figure 2).

156 


\section{DISCUSSION}

158 All preoperative patient-reported factors were more sensitive in detecting in-hospital major morbidity than a high score in either of the ASA scores used in this study. Of all patients with

160

161

162

163

164

165

166

167

168

169

170

171

172

173

174

175

176

177

178

179

180

181

182

183

184

185 major complications, two thirds $(66 \%)$ reported at least one preoperative patient-reported risk factor for adverse outcome. Conversely, the rate of in-hospital major morbidity in patients reporting no preoperative risk factors was only $9 \%$, half of the in-hospital major morbidity rate of $15 \%$ in this cohort. The AUCs of both original ASA score and Helsinki ASA score for adverse outcomes were low. In this study, adverse outcome was defined as major morbidity including both systemic/infectious and neurological complications. In our previous study, we showed an association between a high Helsinki ASA score and systemic/infectious but not neurological complications. The AUCs of our simple composite scores were not perfect, yet superior to those of either of the ASA scores for major morbidity. Thus, combining the patient-reported factors to a composite score to supplement the conventional risk scores is a potential approach to improving the accuracy of risk stratification.

Due to population dynamics, advances in medicine and development of modern surgical techniques, the number of elderly patients is increasing. Over a third of all inpatient surgeries in the US in 2007 were performed on $\geq 65$-year-olds, and the number is expected to double by $2020 .{ }^{19,20}$ Neurosurgery is no exception, as age alone is no longer considered a surgical contraindication. It is known that aging causes many physiological changes and syndromes that lead to increasing fragility, resulting in increased risk of postoperative complications. ${ }^{19,21}$ Conventional risk assessment scores are largely based on the presence of comorbidities and cardiovascular performance, and may overlook subtle geriatric syndromes that translate into increased vulnerability in the elderly, ${ }^{22}$ calling for more refined risk assessment tools in this patient group. Diminished cognitive status was a strong predictor of an adverse postoperative event in our cohort, highlighting the importance of identifying preoperative cognitive dysfunction. This does not require extensive resources, and can be estimated by a short, selffilled questionnaire such as the TYM test in our study. In contrast, perioperative changes in modified Rankin Scale scores seem poorly associated with postoperative complications in elective craniotomy patients. ${ }^{23}$ The Karnofsky Performance Score (KPS), another measure 
commonly used in neurosurgery for assessing the patients' functional capacity, was not preoperatively assessed in our study cohort. Even though our systematic review found support for the use of KPS in the preoperative risk stratification of patients with intracranial tumors, it $\underline{\text { remains unclear whether KPS can reliably predict mortality and morbidity in other patient }}$ groups. ${ }^{11}$

In our cohort advanced age, deteriorating health, and cognitive dysfunction lead not only to increased complication rates but also a different complications profile with a tendency toward major complications. The advantages of our simple composite score of preoperative patientreported factors, compared to either of the ASA scores alone, were especially evident in the subgroup of elderly patients, with an even higher AUC for major morbidity than in the whole cohort. Thus, incorporating patient-reported factors to preoperative risk assessment of the elderly may be advisable, since simple health-related questions are feasibly collected even in the setting of a busy preoperative clinic.

\section{Limitations}

The study has a number of limitations. First, our cohort size was limited considering the low rates of mortality and individual in-hospital complications. The study was conducted in a high-volume tertiary neurosurgical center, and the cohort represents a full year's case mix at our institution. Selection bias cannot be excluded, as the patients with poor health or deprived socioeconomic status are the ones most likely not to respond to questionnaires, ${ }^{24}$ but no major differences were observed in the demographic patient characteristics and surgical indications between the original cohort and the subgroup in this study. Third, inter-rater variability for ASA score and Helsinki ASA score cannot be excluded, but detailed scoring instructions were included in the study protocol to minimize such effect. Limitations of and reasoning behind the used scales have been thoroughly discussed previously. ${ }^{11}$ Finally, there is no universal consensus over categorizing complications in neurosurgical patients. Thus, the inclusion of silent strokes and reoperations in major morbidity may be criticized. We repeated the analyses without these complications to exclude bias in analyses, with unchanged results (results not shown). 


\section{CONCLUSIONS}

216 In conclusion, our results encourage further studies on preoperative patient-reported factors as

217 promising future instruments for improved preoperative risk stratification in neurosurgery.

218 Patient-reported preoperative factors are well suited to guide shared clinical decision-making

219 and to promote patient-centered care. They may also facilitate communication not only

220 between patients and providers but also multidisciplinary teams and improve clinical

221 outcomes and transitions of care. Neurosurgery-specific composites of patient-reported

222 factors may improve the accuracy of conventional risk scores such as the ASA score or the

223 KPS in preoperative risk stratification, especially in elderly patients.

224

225 


\section{REFERENCES}

1. Nashef SA, Roques F, Michel P, Gauducheau E, Lemeshow S, Salamon R. European system for cardiac operative risk evaluation (EuroSCORE). Eur J Cardiothorac Surg. 1999;16:9-13.

2. Nashef SA, Roques F, Sharples LD, et al. EuroSCORE II. Eur J Cardiothorac Surg. Apr 2012;41(4):734-745.

3. American Society of Anesthesiologists. ASA Physical Status Classification System. http://www.asahq.org/resources/clinical-information/asa-physical-status-classificationsystem. Accessed March 21, 2015.

4. Saklad M. Grading of Patients for Surgical Procedures. Anesthesiology. 1941;2:281284.

5. Charlson ME, Pompei P, Ales KL, MacKenzie CR. A New Method of Classifying Prognostic Comorbidity in Longitudinal Studies: A Development and Validation. $J$ Chronic Dis. 1987;40:373-383.

6. Rankin J. Cerebral Vascular Accidents in Patients Over the Age of 60. II. Prognosis. Scott Med J. 1957;2:200-215.

7. van Swieten JC, Koudstaal PJ, Visser MC, Schouten HJ, van Gijn J. Interobserver agreement for the assessment of handicap in stroke patients. Stroke. 1988;19(5):604607.

8. Karnofsky DA, Burchenal JH. The Clinical Evaluation of Chemotherapeutic Agents in Cancer. In: MacLeod CM, ed. Evaluation of Chemotherapeutic Agents. New York, USA: Columbia University Press; 1949:196.

9. Lee TH, Marcantonio ER, Mangione CM, et al. Derivation and Prospective Validation of a Simple Index for Prediction of Cardiac Risk of Major Noncardiac Surgery. Circulation. 1999;100:1043-1049.

10. Goldman L, Caldera DL, Nussbaum SR, et al. Multifactorial index of cardiac risk in noncardiac surgical procedures. $N$ Engl J Med. 1977;297(16):845-850.

11. Reponen E, Tuominen H, Korja M. Evidence for the use of preoperative risk assessment scores in elective cranial neurosurgery: a systematic review of the literature. Anesth Analg. Aug 2014;119(2):420-432. 
12. Reponen E, Korja M, Niemi T, Silvasti-Lundell M, Hernesniemi J, Tuominen H. Preoperative identification of neurosurgery patients with a high risk of in-hospital complications - a prospective cohort of 418 consecutive elective craniotomy patients. J Neurosurg. 2015;123(3):594-604.

13. Reponen E, Tuominen H, Hernesniemi J, Korja M. Patient-reported outcomes in elective cranial neurosurgery. World Neurosurg. 2015;84(6):1845-1851.

14. Reponen E, Tuominen H, Hernesniemi J, Korja M. Patient Satisfaction and ShortTerm Outcome in Elective Cranial Neurosurgery. Neurosurgery. Nov 2015;77(5):769776.

15. Heistaro S. Methodology Report, Health 2000 Survey Helsinki, Finland: National Public Health Institution 2008; http://www.terveys2000.fi/doc/methodologyrep.pdf. Accessed 21 March, 2015.

16. Tan HYF, Aziz AR, Teh KC, Chia YMH. Reliability of the Stair-Climb Test (SCT) of Cardiorespiratory Fitness. Adv Exerc Sports Physiol. 2004;10(3):77-83.

17. Brunelli A, Al Refai M, Monteverde M, Borri A, Salati M, Fianchini A. Stair Climbing Test Predicts Cardiopulmonary Complications After Lung Resection. Chest. 2002;121:1106-1110.

18. Brown J, Pengas G, Dawson K, Brown LA, Clatworthy P. Self administered cognitive screening test (TYM) for detection of Alzheimer's disease: cross sectional study. BMJ. 2009;338:b2030.

19. Etzioni DA, Liu JH, O'Connell JB, Maggard MA, Ko CY. Elderly patients in surgical workloads: a population-based analysis. Am J Surg. 2003;69(11):961-965.

20. Hall MJ, DeFrances CJ, Williams SN, Golosinskiy A, Schwartzman A. National Hospital Discharge Survey: 2007 summary. Natl Health Stat Report. 2010;29:1-20, 24.

21. Turrentine FE, Wang H, Simpson VB, Jones RS. Surgical risk factors, morbidity and mortality in elderly patients. J Am Coll Surg. 2006;203(6):865-877.

22. Kim S, Brooks AK, Groban L. Preoperative assessment of the older surgical patient: honing in on geriatric syndromes. Clin Interv Aging. 2015;10:13-27. 
23. Reponen E, Tuominen H, Hernesniemi J, Korja M. Modified Rankin Scale and ShortTerm Outcome in Cranial Neurosurgery: A Prospective and Unselected Cohort Study. World Neurosurg. Jul 2016;91:567-573 e567.

24. Hutchings A, Neuburger J, Grosse Frie K, Black N, van der Meulen J. Factors associated with non-response in routine use of patient reported outcome measures after elective surgery in England. Health Qual Life Outcomes. 2012;10:34. 
294 Figure 1. ROC curves of orginal ASA score, Helsinki ASA score, and Patient-reported composite score (poor preoperative overall health status, inability to climb two flights of stairs and preoperatively diminished cognitive function) for major morbidity. Abbreviations: ASA, American Society of Anesthesiologists; ROC, receiver-operating characteristics

299

300

Figure 2. ROC curves of original ASA score, Helsinki ASA Score, and patient-reported composite score (poor preoperative overall health status, inability to climb two flights of stairs and preoperatively diminished cognitive function) for major morbidity in patients aged 65 or older. 


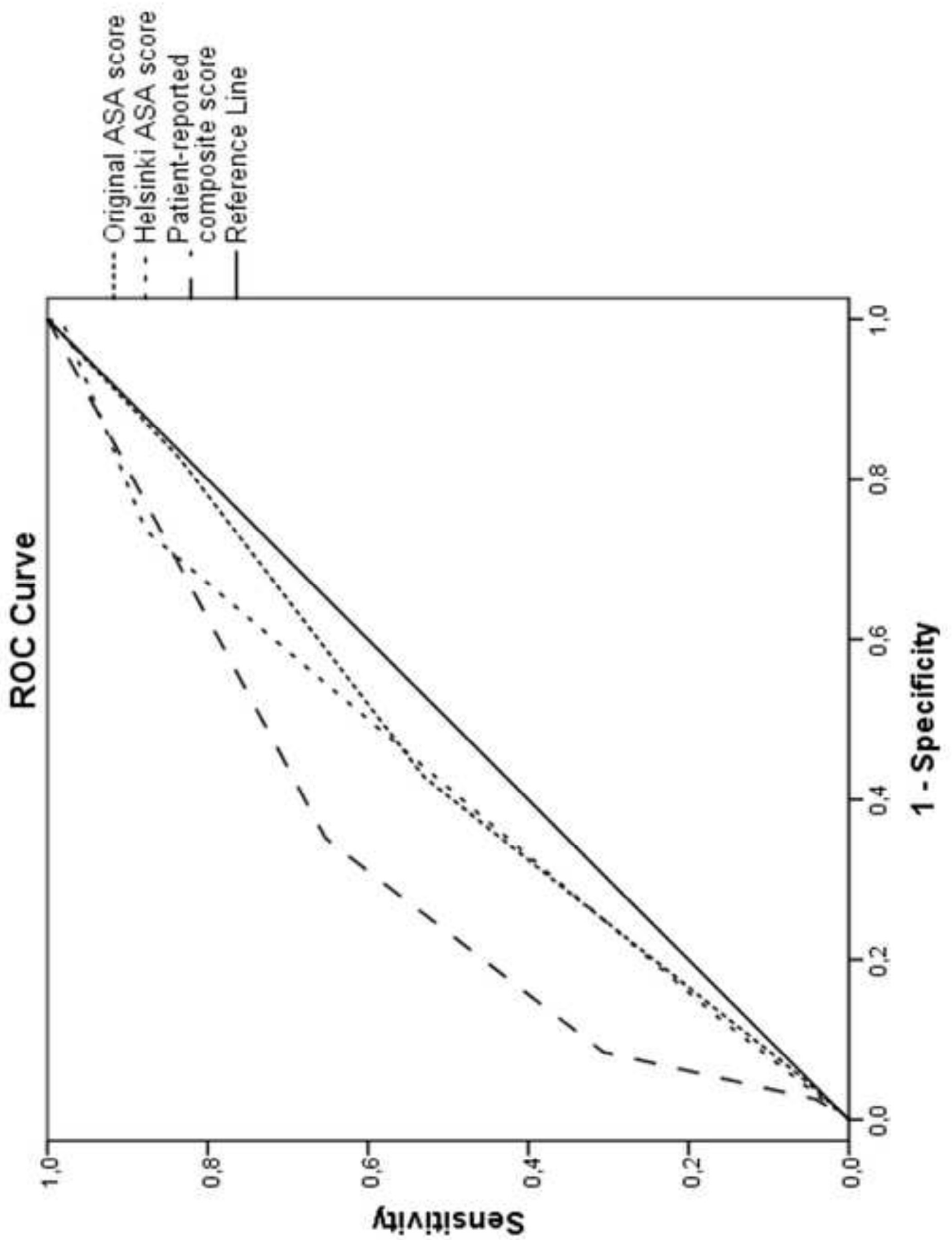




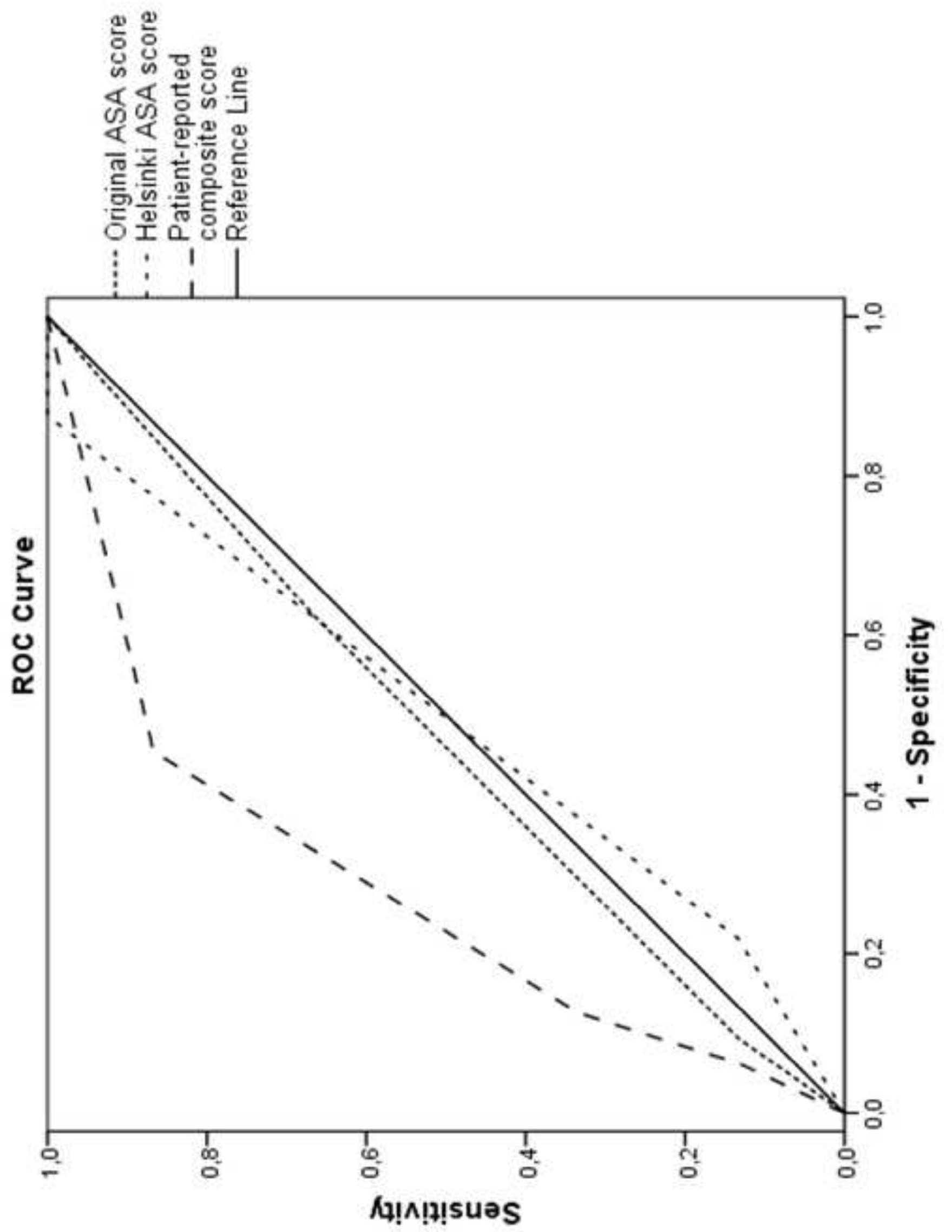


Table 1. Patient characteristics and surgical indications in the original study cohort and the subgroup included in the analyses for patient-reported preoperative variables in risk prediction.

$\begin{array}{ll}\text { Original study cohort } & \text { Subgroup } \\ \mathbf{n}=\mathbf{4 1 8} & \mathbf{n}=\mathbf{3 2 2}\end{array}$

Sex, n (\%)

Male $158(37.8)$

$118(36.6)$

Female

$260(62.2)$

$204(63.4)$

Age (years)

mean (SD), median (range)

56.4 (13.9), 58.0 (18-87)

$55.0(13.5), 57.0(18-83)$

$\geq 65$ years, $n(\%)$

$124(29.7)$

$79(24.5)$

Indication for elective craniotomy, $\mathbf{n}$

(\%)

Vascular lesion

$138(33.0)$

$110(34.2)$

Malignant tumor

$121(28.9)$

$106(32.9)$

Benign tumor

$134(32.1)$

$87(27.0)$

Other

$25(6.0)$

$19(5.9)$ 
Table 2. Percentage distributions of preoperative ASA score, Helsinki ASA and patientreported composite score $(n=322)$

\begin{tabular}{c|c|c|c|} 
& ASA score & $\begin{array}{c}\text { Helsinki } \\
\text { ASA score }\end{array}$ & $\begin{array}{c}\text { Composite } \\
\text { score }\end{array}$ \\
\hline $\mathbf{0}$ & N/A & N/A & 60.2 \\
$\mathbf{1}$ & 17.1 & 0.9 & 28.0 \\
$\mathbf{2}$ & 38.5 & 23.3 & 9.0 \\
$\mathbf{3}$ & 38.8 & 62.4 & 2.8 \\
$\mathbf{4}$ & 5.6 & 13.4 & N/A
\end{tabular}

Abbreviations: ASA, American society of Anesthesiologists; N/A, not applicable.

All emergency (scheduled $<7$ days prior to the surgery) patients were excluded from the study according to the exclusion criteria. Thus, the highest possible ASA score in the cohort was 4 . 
Table 3. Frequencies of individual major complications.

\begin{tabular}{l|c} 
Complication & Number of patients (\%) \\
\hline New or worsened hemiparesis & $28(8.7)$ \\
Re-CRT/EI & $14(4.3)$ \\
Pneumonia & $6(1.9)$ \\
Silent stroke & $3(0.9)$ \\
Mortality (in-hospital) & $2(0.6)$ \\
AMI & $1(0.3)$ \\
DVT & $1(0.3)$ \\
PE & $1(0.3)$ \\
\hline
\end{tabular}

Abbreviations: AMI, acute myocardial infarction; CRT, craniotomy; DVT, deep venous thrombosis; EI, endovascular intervention; PE, pulmonary embolism 
Table 4. Numbers of patients and associations between preoperative patient-reported risk factors, ASA score, and Helsinki ASA score with primary outcomes in univariable analyses. Pearson Chi Square test (categorical) or Mann-Whitney U-test (continuous/ordinal) were used for association analyses. $\mathrm{N}=\mathbf{3 2 2}$, significant associations in bold.

\section{Major in-hospital morbidity}

\begin{tabular}{l|l|l|l|l}
\multicolumn{2}{|l}{} & Yes & No & $\begin{array}{l}\text { p-value } \\
\text { (OR, CI) }\end{array}$ \\
\hline Inability to climb two flights of stairs* & Yes & 12 & 29 & $\begin{array}{l}<\mathbf{0 . 0 1} \\
(\mathbf{2 . 7}, \mathbf{1 . 3}-5.8)\end{array}$ \\
\cline { 2 - 5 } & No & 37 & 244 & \\
\hline Preoperative overall health* & Good & 40 & 249 & $\begin{array}{l}\mathbf{0 . 0 4} \\
(\mathbf{2 . 3}, \mathbf{1 . 0}-5.4)\end{array}$ \\
\cline { 2 - 5 } & Poor & 9 & 24 & \\
\hline Preoperative TYM* & & & & $<\mathbf{0 . 0 1}$ \\
\hline Preoperative ASA score & & & & $0.30^{\dagger}$ \\
\hline Preoperative Helsinki ASA score & & & $0.06^{\dagger}$ \\
\hline
\end{tabular}

*Patient-reported risk factors

${ }^{\dagger} \mathrm{OR}, \mathrm{CI}$ not calculable (continuous/ordinal variables)

Abbreviations: N/A, not applicable; TYM, Test Your Memory -questionnaire; OR, odds ratio; CI, $95 \%$ confidence interval 